Research Article

\title{
Based on M-Copula Reliability Analysis of Random Load Correlation
}

\author{
Huang Bin $\mathbb{D}^{1},{ }^{1}$ Yan Mingdong, ${ }^{1}$ Liu Xiaogang, ${ }^{2}$ and Xiao Mao' \\ ${ }^{1}$ College of Civil Engineering, Xi'an University of Architecture and Technology, Xi'an 710055, Shaanxi, China \\ ${ }^{2}$ Central Research Institute of Building and Construction Co. Ltd. MCC, Beijing 100088, China \\ Correspondence should be addressed to Huang Bin; huangbin@xauat.edu.cn
}

Received 5 January 2020; Revised 11 April 2020; Accepted 12 June 2020; Published 5 August 2020

Academic Editor: Salvatore Caddemi

Copyright (c) 2020 Huang Bin et al. This is an open access article distributed under the Creative Commons Attribution License, which permits unrestricted use, distribution, and reproduction in any medium, provided the original work is properly cited.

Load is one of the main causes of structural failure, and the correlation among loads would affect the evaluation results of structural performance. The purpose of this paper is to analyze the influence of the correlation among multiple loads on the structural reliability. In this paper, the nonparametric kernel density estimation (NKDE) method is used to estimate the probability density function (PDF) of related loads. In addition, the mixed copula (M-Copula) model is proposed, which combines Gumbel copula, Frank copula, Clayton copula, and weight coefficient, and the model parameters are fitted by MATLAB software to get the correlation of related loads. The reliability based on the related load combination is calculated according to the constructed model. After analyzing three numerical cases, the results show that the probability characteristics of NKDE estimation are very close to the actual conditions, and the reliability calculated by the M-Copula model is larger than those calculated by JCSS, Turkstra, and Gong methods. Using the M-Copula model for load correlation would avoid underestimating the reliability of the structure, which is conducive to structural economic development.

\section{Introduction}

With the diversified development of building structure forms, the functions of buildings are becoming more and more comprehensive, which leads to the diversified and complicated load forms in the service cycle of the structure [1-3]. For example, besides common loads, industrial buildings also bear fatigue, impact, additional loads for changing purposes and stacking loads for goods. [4-6]. According these conditions, it is necessary to put forward higher requirements for the load combination method and to consider the complex correlation among various loads in order to ensure economic design of structures and accurately evaluate reliability and safety of structures during service. In the past, random load combination methods mainly included Wen Yi-Kwei [7] coincidence method, upcrossing theory [8], and Kellishen, Ferry-Borges, JCSS rule, and Turkstra combination method commonly used [9]. These methods or theories hold that all loads are independent and unrelated to each other. However, under influence of a certain condition, many loads may change significantly, and in general, there may be no clear correlation between the peak values of load components, but there are synchronous internal relations within the spatial neighborhood. The calculation based on independent variables is obviously unreasonable, which makes calculation results of structural reliability far from reality. At present, many scholars pay more and more attention to the study of load process correlation, such as Gong [9], Li [10], and Yao [11], who have made great contributions to development of load correlation. However, these methods or theories of Gong, Yao, and Li were all based on stationary binomial distribution or decompose loads into independent load pulses. And they emphasize correlation between adjacent loads and consider that correlation between random loads farther apart can be neglected. This idealized treatment is unreasonable, which will affect the structure of structural reliability analysis.

The following is a brief introduction of the commonly used Turkstra method. The core of this method is to find out all variable load effects of structures and set them as the maximum value of the design reference period one by one. 
Other components are combined according to random time value, and results of all the combinations are arranged to find the minimum value, which is taken as the design value of load effect [9]. Assuming that there are $n$ variable loads participating in combination, the stochastic function of effect can be expressed as $\left\{S_{i}(t), t \in[0, T]\right\},(i=1,2, \ldots, n)$. So, they have $n$ maximum combinations and can be expressed as [12]

$$
\begin{array}{r}
S_{M 1}=\max _{0 \leq t \leq T} S_{1}(t)+S_{2}\left(t_{0}\right)+\cdots+S_{n}\left(t_{0}\right), \\
S_{M 2}=S_{1}\left(t_{0}\right)+\max _{0 \leq t \leq T} S_{2}(t)+\cdots+S_{n}\left(t_{0}\right), \\
\cdots \cdots, \\
S_{M n}=S_{1}\left(t_{0}\right)+S_{2}\left(t_{0}\right)+\cdots+\max _{0 \leq t \leq T} S_{n}(t),
\end{array}
$$

where $S_{M i}$ is maximum value of the $i$ combination. This combination method is simple in construction, which considered that variable loads are independent of each other. It only needs to list all the combination forms one by one and calculate the maximum value of each combination separately. However, this method is not necessarily the same as actual load combination, such as twin load [9, 13]. The results of this calculation method are quite erroneous.

In recent studies, copula function has been widely used in constructing multidimensional joint distribution functions. In view of the problems of load correlations, this paper proposes a M-Copula model of multivariate complex loads. The advantage of this model is that it can flexibly establish the joint distribution function of multivariate random loads and is not affected by the probability density function of single load.

\section{Load Effect Combination Method Based on Copula Function}

2.1. Theory of Copula Function. After Sklar [14] proposed copula function in 1959, it was successfully applied in fields of statistical economics and finance [15], hydrology [16-18], and structural engineering [19-24], and a lot of research papers were published. The advantage of copula function is that it can directly construct joint distribution function for many related random variables or decouple a joint distribution function into multiple marginal distribution functions and a unique copula function [25]. There are more than twenty copula functions commonly used, such as Gauss copula, Gumbel copula, Clayton copula, and Frank copula. The copula functions selected and constructed are different, and joint distribution functions are also different. According to Sklar theorem [14], for $n$ consecutive marginal cumulative distribution functions $F_{1}, F_{2}, \ldots, F_{n}$, there is only one $n$ dimensional copula function $C(\cdot)$, so that the following equation holds

$$
F\left(x_{1}, x_{2}, \ldots, x_{n}\right)=C\left(F_{1}\left(x_{1}\right), F_{2}\left(x_{2}\right), \ldots, F_{n}\left(x_{n}\right)\right),
$$

where $F\left(x_{1}, x_{2}, \ldots, x_{n}\right)$ is the joint distribution function of the random vector $\mathbf{x}=\left\{x_{1}, x_{2}, \ldots, x_{n}\right\}, C(\cdot)$ is the copula function, and $F_{i}\left(x_{i}\right)$ is marginal distribution function of $x_{i}$. Therefore, seemingly unrelated single-load random variables can establish some relationship according to equation (2), and any change of random variables may affect results of combination. The joint probability density function of the random vector $\mathbf{x}=\left\{x_{1}, x_{2}, \ldots, x_{n}\right\}$ can be obtained by calculating the derivative of equation (2). The process is as follows:

$$
\frac{\partial^{n}\left[F\left(x_{1}, x_{2}, \ldots, x_{n}\right)\right]}{\partial x_{1} \partial x_{2} \ldots \partial x_{n}}=\frac{\partial^{n}\left[C\left(F_{1}\left(x_{1}\right), F_{2}\left(x_{2}\right), \ldots, F_{n}\left(x_{n}\right)\right)\right]}{\partial x_{1} \partial x_{2} \ldots \partial x_{n}} \text {. }
$$

Equation (3) is simplified to

$$
f\left(x_{1}, x_{2}, \ldots, x_{n}\right)=c\left(F_{1}\left(x_{1}\right), F_{2}\left(x_{2}\right), \ldots, F_{n}\left(x_{n}\right)\right) \prod_{i=1}^{n} f_{i}\left(x_{i}\right) \text {, }
$$

where $c\left(u_{1}, u_{2}, \ldots, u_{n}\right)=\left(\partial^{n} C\left(u_{1}, u_{2}, \ldots, u_{n}\right) / \partial u_{1} \partial u_{2} \ldots \partial_{n}\right)$, $u_{i}=F_{i}\left(x_{i}\right), f_{i}\left(x_{i}\right)$ is marginal probability density function of $x_{i}$, and $f\left(x_{1}, x_{2}, \ldots, x_{n}\right)$ is the joint probability density function of $\mathbf{x}=\left\{x_{1}, x_{2}, \ldots, x_{n}\right\}$.

In order to study relationship between loads, it is necessary to select a copula function. The Gaussian copula function is a copula function of multivariate normal distribution. Its advantage is that it can construct correlation function between multiple random variables [14] and representation as

$$
C_{R}^{n}\left(u_{1}, u_{2}, \ldots, u_{n}\right)=\Phi_{R}\left(\varphi^{-1}\left(u_{1}\right), \varphi^{-2}\left(u_{2}\right), \ldots, \varphi^{-1}\left(u_{n}\right)\right),
$$

where $\Phi_{R}(\cdot)$ is standard Gaussian distribution, $R$ is correlation coefficient matrix, and $\varphi^{-1}(\cdot)$ is inverse function of standard Gaussian distribution. When calculating random variables with the degree of freedom $n$, there exists $\mathrm{n}$-fold integral in Gaussian copula function, which is inconvenient for digital calculation. Considering feasibility of calculation, Gumbel copula function, Frank copula function, and Clayton copula function belong to the Archimedean copulas family can be used. They have obvious computing advantages: calculation process is relatively simple and high accuracy, so they are widely used in practical engineering.

If there is a generator $\phi_{\alpha}(u)=-(-\ln u)^{\alpha}$, the Gumbel copula function can be deduced as $C_{\alpha}^{\text {Gumbel }}(u)$ or denoted as $C^{\text {Gumbel }}(u, \alpha)$, and the calculation method be expressed as

$$
\begin{aligned}
& C^{\text {Gumbel }}\left(u_{1}, u_{2}, \ldots, u_{n} ; \alpha\right) \\
& \quad=\exp \left\{-\left[\left(-\ln u_{1}\right)^{\alpha}+\left(-\ln u_{2}\right)^{\alpha}+\cdots+\left(-\ln u_{n}\right)^{\alpha}\right]^{1 / \alpha}\right\},
\end{aligned}
$$

where $\alpha$ is the parameter of Gumbel copula function and $\alpha$ range of which is $[1, \infty)$, and the change of $\alpha$ values will affect the correlation degree of random variables. If the value of $\alpha$ is 1 , then the random variables $u_{1}, u_{2}, \ldots, u_{n}$ are independent of each other; if the value range of $\alpha$ approaches $+\infty$, then the random variables $u_{1}, u_{2}, \ldots, u_{n}$ are completely correlated [14].

If there is a generator $\phi_{\beta}(u)=-\ln [(\exp (-$ $\beta u)-1) /(\exp (-\beta)-1)]$, the Frank copula function can be deduced as $C_{\beta}^{\text {Frank }}(u)$ or denoted as $C^{\text {Frank }}(u ; \beta)$, and the calculation method be expressed as 


$$
\begin{aligned}
& C^{\text {Frank }}(u ; \beta) \\
& \quad=-\frac{1}{\beta} \ln \left\{1+\frac{\left[\exp \left(-\beta u_{1}\right)-1\right] \ldots\left[\exp \left(-\beta u_{n}\right)-1\right]}{\exp (-\beta)-1}\right\},
\end{aligned}
$$

where $\beta$ is the parameter of Frank copula function and $\beta$ range of which is $(-\infty, 0) \cup(0,+\infty)$, and change of $\beta$ values will affect correlation degree of random variables. Similarly, if there is a generator $\phi_{\gamma}(u)=(1 / \gamma)\left(u^{-\gamma}-1\right)$ and the range of $\gamma$ is $(0,+\infty)$, the Clayton copula function can be deduced as $C_{\gamma}^{\text {Clayton }}(u)$ or denoted as $C^{\text {Clayton }}(u ; \gamma)$, and the calculation method be expressed as

$$
\begin{aligned}
& C^{\text {Clayton }}\left(u_{1}, u_{2}, \ldots, u_{n} ; \gamma\right) \\
& \quad=\max \left\{\left[\left(u_{1}^{\gamma}+u_{2}^{\gamma}+\cdots+u_{n}^{\gamma}\right)-1\right]^{-1 / \gamma}, 0\right\} .
\end{aligned}
$$

Assuming the two-dimensional random variables $\left(U_{1}, U_{2}\right)$, the values of $(\alpha, \beta, \gamma)$ are 1,5 , and 10 , respectively, to generate 2000 random numbers. The scatter plot is shown in Figure 1.

Figure 1 shows that different copula functions have their own distinct characteristics. Using a single copula function cannot accurately describe random characteristics of complex loads, so it is necessary to synthesize a variety of copula functions for analysis.

2.2. Copula Model of Load Combination. Load action is an unstable process that changes with time, but the law of development is not uncorrelated [9-11, 26]. The model of load effect combination can well reflect the correlation between loads. Total load effect is results of multiple loads. Most of loads have strong or weak correlation. Although the load combination model has obtained a lot of research results, most of them focus on the study of correlation of twodimensional loads. The combination model of three-dimensional loads and more than three-dimensional loads is not perfect, which is not conducive to development of load correlation research. The $\mathrm{M}$-Copula function presented below can be used to establish a multiload correlation model, which provides a feasible method for the study of the correlation between multiloads.

2.2.1. Creating M-Copula Model. According to the basic process of the Turkstra method, in the service period $[0, T]$, load stochastic process is divided into $k$ segments on average, and each calculation time is often $\tau$; the length of $j$ segment is $\tau_{j}$; in any period of time interval $\tau_{j}$, the probability of load occurrence is $p_{j}$, and then $q_{j}=1-p_{j}$. If the average score of load stochastic process is enough, then the probability density function of loads in $\left[\tau_{j^{-}}, \tau_{j^{+}}\right]$time is consistent; that is, $f_{X}^{\tau_{j^{-}}}(x, t)=f_{X}^{\tau_{j^{+}}}(x, t)$. In addition, another important assumption of the Turkstra method is that load effects in each time period are independent of each other. However, the load effect is a continuous function, and it is the result of the combination of various random loads, so the results of the Turkstra combination method are not accurate enough.
Copula function can solve problems of load dependence in each time period. Similarly, the discretization method is used. If there are $n$ loads and they are correlated with each other, then the total load effect $S$ can be expressed as

$$
\mathbf{S}=\left[\mathbf{S}_{1}, \mathbf{S}_{2}, \ldots, \mathbf{S}_{\mathbf{n}}\right]^{T}=\left[\begin{array}{ccc}
S_{1\left(\tau_{1}\right)} & \ldots & S_{1\left(\tau_{k}\right)} \\
\ldots & \ldots & \ldots \\
S_{n\left(\tau_{1}\right)} & \ldots & S_{n\left(\tau_{k}\right)}
\end{array}\right]
$$

Load $S_{i}(i=1,2, \ldots, n)$ is also decomposed into $k$ segments, and each calculation time is $\tau_{j}$. The specific process can be shown in Figure 2 .

When load effect is discretized, the length of the $j$-th period is $\tau_{j}$, and the load effect is $S_{i\left(\tau_{j}\right)}$. Then, load effect is composed of $k$ load effect, which can be expressed as a function of $k$-dimensional random variables:

$$
\mathbf{S}_{i}=\left(S_{i\left(\tau_{1}\right)}, S_{i\left(\tau_{2}\right)}, \ldots, S_{i\left(\tau_{k}\right)}\right)
$$

Assuming that probability density function of the $i$ load effect is $f\left(s_{i}\right), C\left(u_{1}, u_{2}, \ldots, u_{n}\right)$ can be calculated according to equation (4), but because of complexity of load correlation and diversity of load probability distribution, it is impossible to accurately simulate the correlation with a single copula function, so a hybrid copula function modeling method is proposed. In this paper, a new M-Copula load combination method is constructed by Gumbel copula function, Frank copula function, Clayton copula function, and some weights, which can be expressed by

$$
\begin{aligned}
C_{\text {mix }} & \left(u_{1}, u_{2}, \ldots, u_{n} ; \omega_{1}, \omega_{2}, \omega_{3}, \alpha, \beta, \gamma\right) \\
= & \omega_{1} C^{G}\left(u_{1}, u_{2}, \ldots, u_{n} ; \alpha\right)+\omega_{2} C^{F}\left(u_{1}, u_{2}, \ldots, u_{n} ; \beta\right) \\
& +\omega_{3} C^{C}\left(u_{1}, u_{2}, \ldots, u_{n} ; \gamma\right),
\end{aligned}
$$

where $C_{\text {mix }}(\cdot)$ is mixed copula function, $\left(C^{G}, C^{F}, C^{C}\right)$ are expressed as Gumbel copula function, Frank copula function, and Clayton copula function, respectively, $\omega_{i}$ is weight coefficient, and $\sum_{i=1}^{n} \omega_{i}=1,(\alpha, \beta, \gamma)$ are correlation degree of random variable $u_{1}, u_{2}, \ldots, u_{n}$. By establishing the M-Copula correlation model, the results of load effect combination can fully reflect relationship between loads and obtain more accurate reliability calculation results.

\subsubsection{Nonparametric Kernel Density Function Estimation.} Load effect varies with time, and it is also a comprehensive response under various loads. If structures encounter ultimate loads during its service period, structures will be in a dangerous ultimate state. In this case, it is impossible to estimate probability density function of a single load. In other words, probability density function of a single load decoupled from presupposed probability density function under combined action of multiple loads will no longer be accurate. In this case, the nonparametric kernel density function estimation method [27-30] should be used to reconstruct the edge probability density function of a certain load. In order to analyze correlation of load random variables, the $n$-dimensional random variables $\left(u_{1}, u_{2}, \ldots, u_{n}\right)$ 


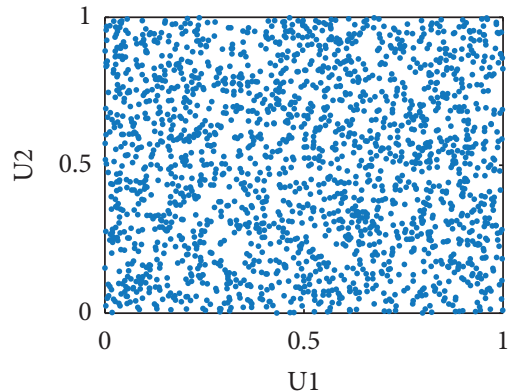

$\mathrm{U} 1$

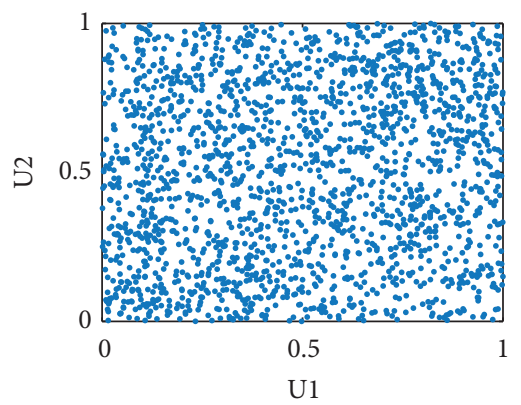

U1

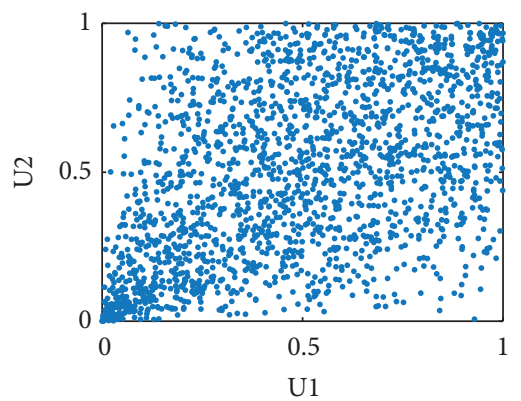

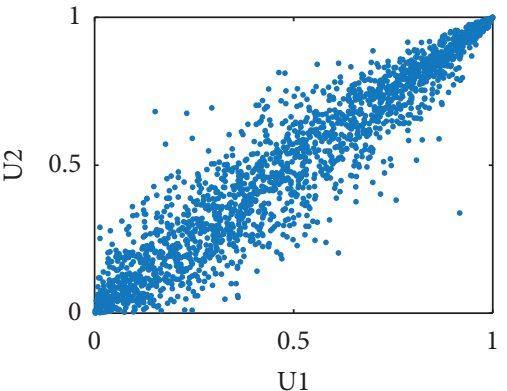

(a)
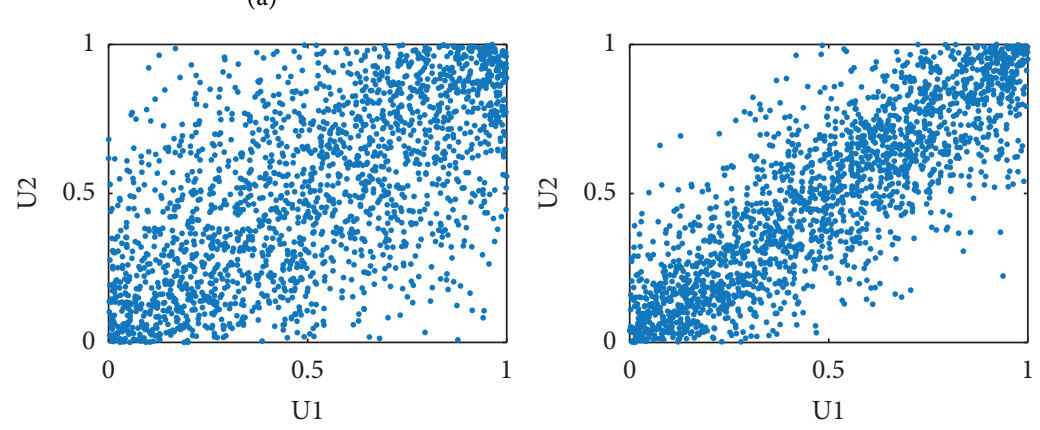

(b)
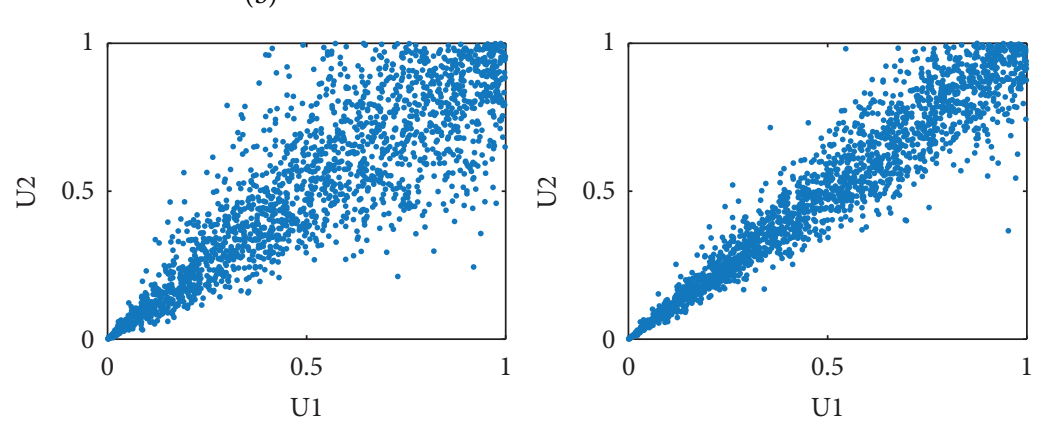

(c)

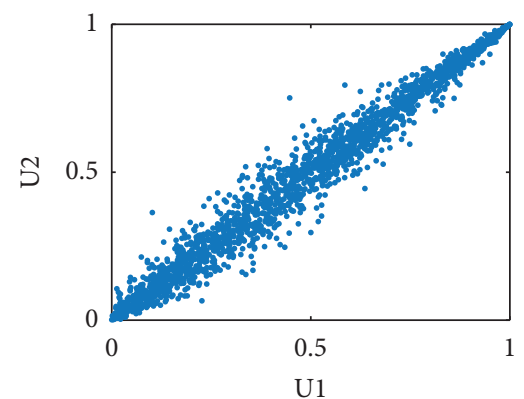

Figure 1: Scatter plot. (a) Gumbel copula $(\alpha=1,5,10)$. (b) Frank copula $(\beta=1,5,10)$. (c) Clayton copula $(\gamma=1,5,10)$.

are analyzed, and combination equations (4) and (11) show that

$$
\begin{aligned}
& F\left(x_{1}, x_{2}, \ldots, x_{n}\right) \\
& \quad=C\left(F\left(x_{1}\right), F\left(x_{2}\right), \ldots, F\left(x_{n}\right)\right)=C\left(u_{1}, u_{2}, \ldots, u_{n}\right),
\end{aligned}
$$

and $\quad c\left(u_{1}, u_{2}, \ldots, u_{n}\right)=\left(\partial^{n} C\left(u_{1}, u_{2}, \ldots, u_{n}\right) / \partial u_{1} \partial u_{2} \ldots\right.$ $\left.\partial u_{n}\right)$. If there are variable matrix $\mathbf{u}$, when the number of $n$ and $h_{n}$ is large enough, $n$ and $h_{n}$ are correlated with each other, and $h_{n}>0$, then the kernel density estimate of $\mathbf{u}$ can be expressed as follows:

$$
\widehat{f}_{\mathbf{u}}(\mathbf{u})=\frac{1}{n h_{n}} \sum_{i=1}^{n} K\left(\frac{u_{i}-\mathbf{u}}{h_{n}}\right),
$$

where $K(\cdot)$ is kernel function, $h_{n}$ is bandwidth, and $\mathbf{u}=\left(u_{1}, u_{2}, \ldots, u_{i}\right)$. Set $u_{i}$ is divided into $k$ time periods and the $j$-th interval is $\tau_{j}$, then probability density function of the scorable quantity $u_{i}$ can be expressed as

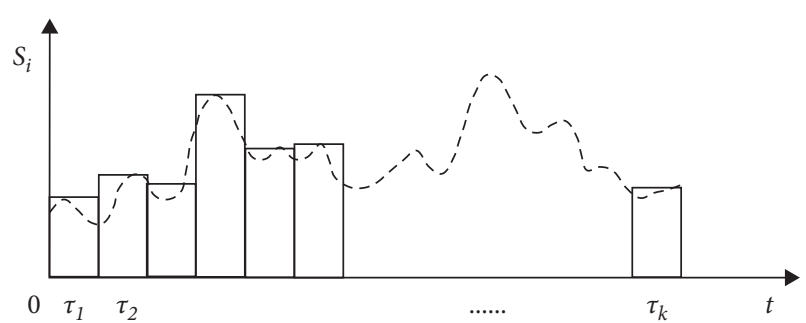

Figure 2: Random process chart of load effects.

$$
\widehat{f}_{u_{i}}\left(u_{1}\right)=\frac{1}{k \tau_{j}} \sum_{j=1}^{k} K\left(\frac{u_{i j}-\mathbf{u}_{i}}{\tau_{j}}\right)=\frac{1}{k \tau} \sum_{j=1}^{k} K\left(u_{i j}-\mathbf{u}_{i}\right),
$$

where Kernel function $\left[K_{u_{1}}(\cdot), K_{u_{2}}(\cdot), \ldots, K_{u_{n}}(\cdot)\right]$ can generally be determined by the empirical method, and bandwidth $\left(\tau_{u_{1 j}}, \tau_{u_{2 j}}, \ldots, \tau_{u_{n i}}\right)$ can take different values. When $n \longrightarrow \infty$ or $\quad\left(1 / k \tau_{j}\right) \longrightarrow 0, \quad\left[\widehat{f}_{u_{1}}\left(u_{1}\right), \widehat{f}_{u_{2}}\left(u_{2}\right)\right.$ $\left., \ldots, \widehat{f}_{u_{n}}\left(u_{n}\right)\right]\left[f_{u_{1}}\left(u_{1}\right), f_{u_{2}}\left(u_{2}\right), \ldots, f_{u_{n}}\left(u_{n}\right)\right]$. In order to 
ensure accuracy of results, the number of simulations $k$ is as much as possible. In the case of the large sample, Gauss distribution can be chosen as kernel function of the kernel density estimation function and can be expressed as [31]

$$
K_{u_{i}}\left(\frac{u_{i j}-\mathbf{u}_{i}}{\tau_{u_{\mathrm{ij}}}}\right)=\frac{1}{\sqrt{2 \pi}} \exp \left[-\frac{\left(u_{i j}-\mathbf{u}_{i}\right)^{2}}{2 \tau_{i j}^{2}}\right],
$$

where $i=1,2, \ldots, n$ and $j=1,2, \ldots, k$. After obtaining kernel function, marginal probability density function $\left[f_{u_{1}}\left(u_{1}\right), f_{u_{2}}\left(u_{2}\right), \ldots, f_{u_{n}}\left(u_{n}\right)\right]$ and marginal distribution function $\left[F_{u_{1}}\left(u_{1}\right), F_{u_{2}}\left(u_{2}\right), \ldots, F_{u_{n}}\left(u_{n}\right)\right]$ of each variable of matrix variable $\mathbf{u}=\left(u_{1}, u_{2}, \ldots, u_{i}\right)$ can be obtained according to equation (15).

2.2.3. Multivariate Sequence Correlation Analysis. If there are $n$ variables $\mathbf{X}=\left(X_{1}, X_{2}, \ldots, X_{n}\right)$, then $\left(x_{1, i-1}, x_{2, i-1}, \ldots\right.$, $\left.x_{n, i-1}\right)$ and $\left(x_{1, i}, x_{2, i}, \ldots, x_{n, i}\right)$ are the $i-1$ and $i$ components of variable $\boldsymbol{X}$. Assuming that marginal distribution function of each component is $\left(F\left(x_{1,1}\right), \ldots, F\left(x_{1, k}\right)\right) \ldots\left(F\left(x_{n, 1}\right), \ldots\right.$,
TABLE 1: Relation between copula function parameters and Kendall's rank correlation coefficient.

\begin{tabular}{lc}
\hline $\begin{array}{l}\text { Copula } \\
\text { functions }\end{array}$ & $\begin{array}{c}\text { The calculation method of mutual transformation } \\
\text { between } \rho \text { and }(\alpha, \beta, \gamma)\end{array}$ \\
\hline $\begin{array}{l}\text { Gumbel } \\
\text { copula }\end{array}$ & $\rho=(1-1 / \alpha)$ \\
$\begin{array}{l}\text { Frank } \\
\text { copula }\end{array}$ & $\rho=1+(4 / \beta)\left[(1 / \beta) \int_{0}^{n}\left(t / e^{t}-1\right) d t-1\right]$ \\
$\begin{array}{l}\text { Clayton } \\
\text { copula }\end{array}$ & $\rho=(\gamma /(2+\gamma))$
\end{tabular}

$\left.F\left(x_{n, k}\right)\right)$ unchanged, it can be expressed as $\left[F\left(X_{1}\right), \ldots\right.$, $\left.F\left(X_{n}\right)\right]$. If $\left[\left(x_{1,1}, x_{2,1}, \ldots, x_{n, 1}\right),\left(x_{1,2}, x_{2,2}, \ldots, x_{n, 2}\right), \ldots\right.$, $\left.\left(x_{1, k}, x_{2, k}, \ldots, x_{n, k}\right)\right]$ are randomly generated samples, the joint distribution function of variables $\boldsymbol{X}$ can be expressed as $H\left(X_{1}, X_{2}, \ldots, X_{n}\right)$, and the corresponding copula function is $C\left(X_{1}, X_{2}, \ldots, X_{n}\right)$, then Kendall's rank correlation coefficient $\hat{\rho}$ of variable $\left(X_{1}, X_{2}, \ldots, X_{n}\right)$ can be expressed as [31]

$$
\widehat{\rho}\left(X_{1}, X_{2}, \ldots, X_{n}\right)=\frac{\sum_{i=1}^{n} \sum_{j=1}^{n} \hat{\rho}\left(X_{i}, X_{j}\right)}{(n(n-1) / 2)} \quad(1 \leq i \leq n, 1 \leq j \leq k, i \neq j),
$$

where $\hat{\rho}\left(X_{1}, X_{2}, \ldots, X_{n}\right)$ are Kendall's rank correlation coefficient of the whole sample of variables $\mathbf{X}, \hat{\rho}\left(X_{i}, X_{j}\right)$ are the Kendall's rank correlation coefficient of the sample of variables $\left(X_{i}, X_{j}\right), n$ is the degree of freedom of random variables, and $k$ is the number of the random sample. According to the simple algorithm, the calculation method of Kendall's rank correlation coefficient $\hat{\rho}\left(X_{i}, X_{k}\right)$ of any two random variables can be as follows:

$$
\hat{\rho}\left(X_{i}, X_{v}\right)=\frac{2}{k(k-1)} \sum_{1 \leq \xi \leq \eta \leq k} \operatorname{sign}\left(X_{i, \xi}-X_{i, \eta}\right)\left(X_{v, \xi}-X_{v, \eta}\right),
$$

where $\hat{\rho}\left(X_{i}, X_{v}\right)$ are Kendall's rank correlation coefficient of any two variables, and then $1 \leq i \leq v \leq n$ and $1 \leq \xi \leq \eta \leq k$; among them,

$$
\operatorname{sign}\left(X_{i, \xi}-X_{i, \eta}\right)\left(X_{v, \xi}-X_{v, \eta}\right)=\left\{\begin{array}{l}
1,\left(X_{i, \xi}-X_{i, \eta}\right)\left(X_{v, \xi}-X_{v, \eta}\right)>0 \\
0,\left(X_{i, \xi}-X_{i, \eta}\right)\left(X_{v, \xi}-X_{v, \eta}\right)=0 \\
-1,\left(X_{i, \xi}-X_{i, \eta}\right)\left(X_{v, \xi}-X_{v, \eta}\right)<0
\end{array}\right.
$$

The Kendall's rank correlation coefficients of any two variables can be obtained by equation (18), and sample estimates of Kendall's rank correlation coefficients $\hat{\rho}$ of population variables can be calculated by equation (16), from which $\hat{\rho}\left(X_{1}, X_{2}, \ldots, X_{n}\right)=\rho\left(X_{1}, X_{2}, \ldots, X_{n}\right)$ can be obtained approximately. According to Table 1, the Kendall's rank correlation coefficient and copula function parameters can be transformed.

The weight coefficient $\omega_{i}$ is estimated by the maximum likelihood estimation method [24]. Based on the M-Copula load correlation analysis model, the specific process is divided into the following steps:
(1) Estimate the number of relevant loads of structures in the service period $[0, T]$, divide it into $n$ time intervals, the $i$ time interval is $\tau_{i}$, and $i=1,2, \ldots, n$.

(2) According to the sample dates of random load $S_{i}$ in the $\tau_{i}$ interval of each period and denoted $S_{i j}$, $j=1,2, \ldots, k$.

(3) The Kendall's rank correlation coefficients of any two kinds of related loads are calculated, and then the Kendall's rank correlation coefficients and the parameters of copula function are estimated. 
(4) By using the maximum likelihood estimation method, weight parameters $\left(\omega_{1}, \omega_{2}, \omega_{3}\right)$ are estimated, and load correlation analysis model $C\left(\alpha, \beta, \gamma ; \omega_{1}, \omega_{2}, \omega_{3}\right)$ based on M-Copula function is obtained. Therefore, it is expressed in Figure 3.

\section{Reliability Calculation Model Based on M-Copula}

The traditional reliability calculation method is probability expression of resistance and load, which is independent when dealing with loads and does not consider the complex correlation between loads, so failure probability obtained by the general calculation method is inconsistent with actual situation. Considering the correlation of various loads will ultimately affect reliable calculation results. Different results of load combination will affect results of reliability calculation. The concept of reliability is probability of completing a predetermined function within a specified time under specified conditions. In order to obtain reliable probability, it is necessary to calculate load effect and resistance. If the structure is in a good working environment, it can be approximated that resistance of the structure will not change during service, but this assumption is unreasonable, because structures will suffer uncertain damages during service, which will greatly affect the performance of the structure.

Similarly to the principle of Figure 2, the resistance of structures can be decomposed into $k$ time intervals during the whole service period $[0, T]$, and resistance value in the $j$ th period is $R_{j}$, as shown in Figure 4 .

If the number of $\tau_{j}$ or $k$ is large enough, it can be considered that $R_{j\left(\tau_{i}\right)}$ does not change in the time range of $\tau_{j}$, and then the reliability model can be expressed as

$$
\begin{aligned}
P_{f}= & P(Z<0)=P\left(\min R-\sum_{i=1}^{n} S_{i}\right), \\
= & P\left\{\left[\left(\min R-S_{1}\right)<0\right] \cup\left[\left(\min R-S_{2}\right)<0\right]\right. \\
& \cdot \cup \ldots \cup[(\min R-S)<0]\},
\end{aligned}
$$

where $Z$ is performance function, $S_{\tau_{i}}$ is load effect in the time range of $\tau_{i}$, and $\min (\cdot)$ is small value function. $P_{f}$ represents failure probability of structures. For $n$-related loads, the failure probability of structures can be expressed as

$$
\begin{aligned}
P_{f}(Z) & =P(Z<0)=P\left[\left(\min R-C\left(u_{1}, u_{2}, \ldots, u_{n}\right)\right)<0\right], \\
& =P\left[\left(\min R-C\left(u_{1}, u_{2}, \ldots, u_{n} ; \alpha, \beta, \gamma ; \omega_{1}, \omega_{2}, \omega_{3}\right)\right],\right.
\end{aligned}
$$

where $(\alpha, \beta, \gamma)$ are parameters of copula function and $\left(\omega_{1}, \omega_{2}, \omega_{3}\right)$ are weight coefficient.

\section{Case Analysis}

The actual loads suffered by structures usually have many forms, such as Gauss distribution (GD) and Gumbel distribution (Gumbel I). In order to reflect correlation analysis of multidimensional correlated loads, the three-dimensional load correlation is selected to study. Then, the M-Copula model can be expressed as

$$
\begin{aligned}
C_{\text {mix }} & \left(u_{1}, u_{2}, u_{3} ; \omega_{1}, \omega_{2}, \omega_{3}, \alpha, \beta, \gamma\right) \\
= & \omega_{1} C^{G}\left(u_{1}, u_{2}, u_{3} ; \alpha\right)+\omega_{2} C^{G}\left(u_{1}, u_{2}, u_{3} ; \beta\right) \\
& +\omega_{3} C^{G}\left(u_{1}, u_{2}, u_{3} ; \gamma\right) .
\end{aligned}
$$

Next, through analysis of specific statistical dates, the confidence level is 95\%. Figure 5 shows sample dates of actual statistical results of loads $\left(S_{1}, S_{2}, S_{3}\right)$ and results of NKDE fitting.

From the results of Figure 5, it is found that the three load effects are not completely random but conform to some form of distribution. The fitting distribution parameters are shown in Table 2.

By comparing the results of the above NKDE estimation method with actual data, it is found that probability density function obtained by the nonparametric kernel density estimation method has a good accuracy, which will be conducive to calculation of reliability. Common variable loads are usually distributed in extreme form. Load effects are combined on basis of Ferry-Borges, JCSS, and Turkstra combination methods commonly used in the past. The basic core of these methods is to determine maximum value in the time interval and then take the basic time point value as combination value or use each time interval. The maximum value in the interval is combined, which is obviously too safe. At the same time, it will lead to the high reliability design value of structure, which is not conducive to economic design of structures.

However, probabilistic density function of nonparametric kernel density estimation can weaken this effect from each component of load effect combination, and calculation results are reliable. According to the same method, other two groups of data samples are analyzed, and the distribution parameters of three groups of data are finally expressed in Table 3.

According to equation (16) and Table 1, the parameters of Gumbel copula, Frank copula, and Clayton copula functions can be estimated by using MATLAB R2014b programming. The estimated results are expressed in Table 4.

According to the results of parameter estimation, the M-Copula model established in this paper is used to calculate structural reliability index $R_{\mathrm{el}}$, and the results are compared with those calculated by JCSS, Turkstra, and Gong methods. Comparison results are shown in Table 5. Calculation results in Table 5 show that correlation between complex loads will affect the calculation results of reliability and even change evaluation of structural performance. It is pointed out in the table that traditional JCSS and Turkstra methods are obviously conservative in calculating reliability evaluation indicators, which will inevitably lead to uneconomical treatment schemes. The M-Copula function model presented in this paper can well describe relationship between multiple complex loads, accurately calculate reliability index, and provide an accurate and scientific method for structural performance evaluation. 


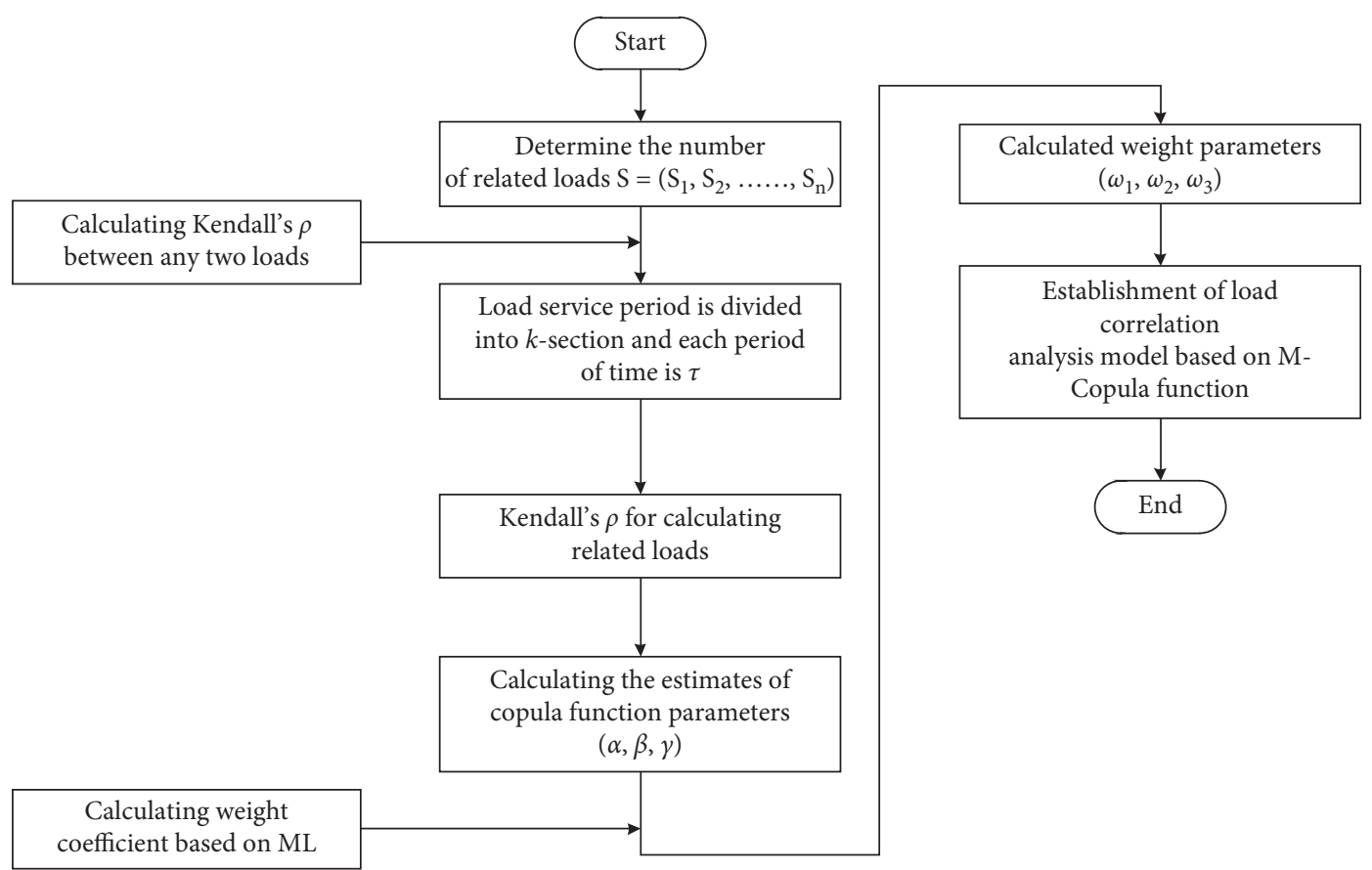

Figure 3: Flowchart of the M-Copula function model.

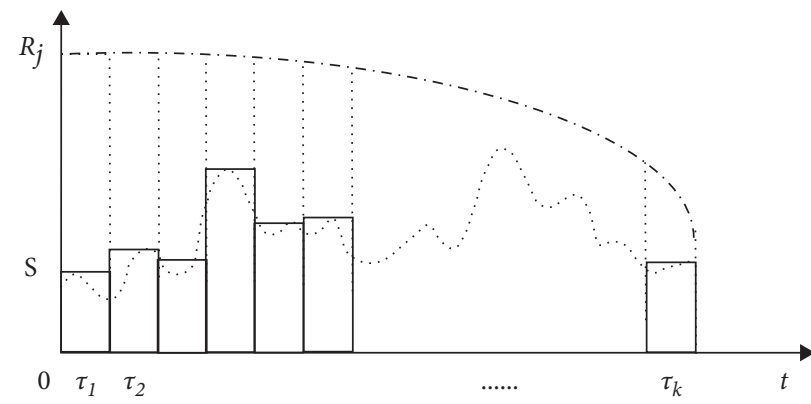

Figure 4: Time-varying load and resistance model.

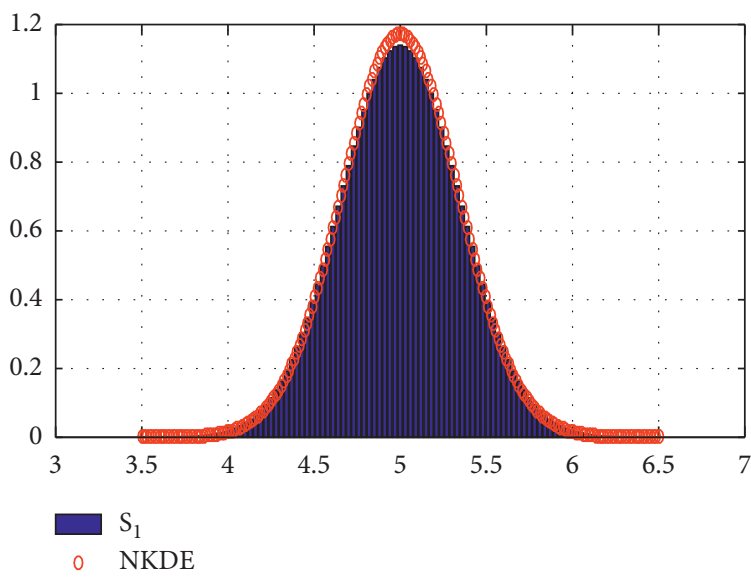

(a)

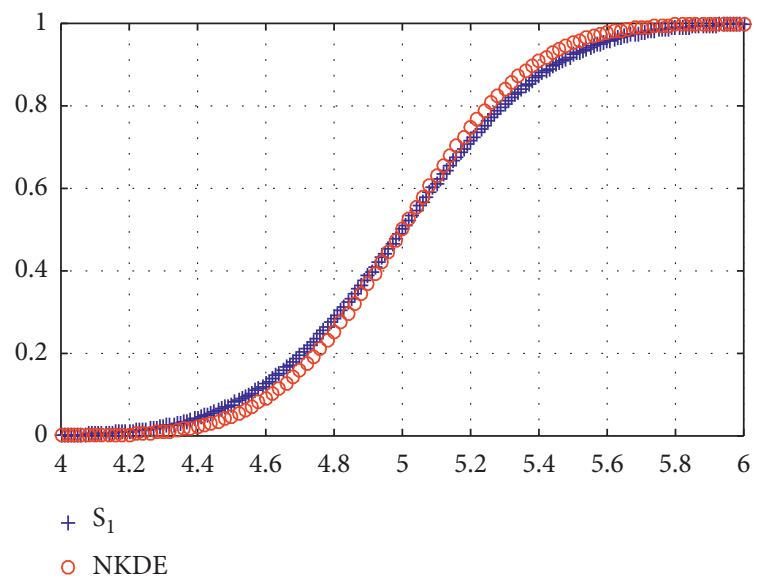

(b)

Figure 5: Continued. 


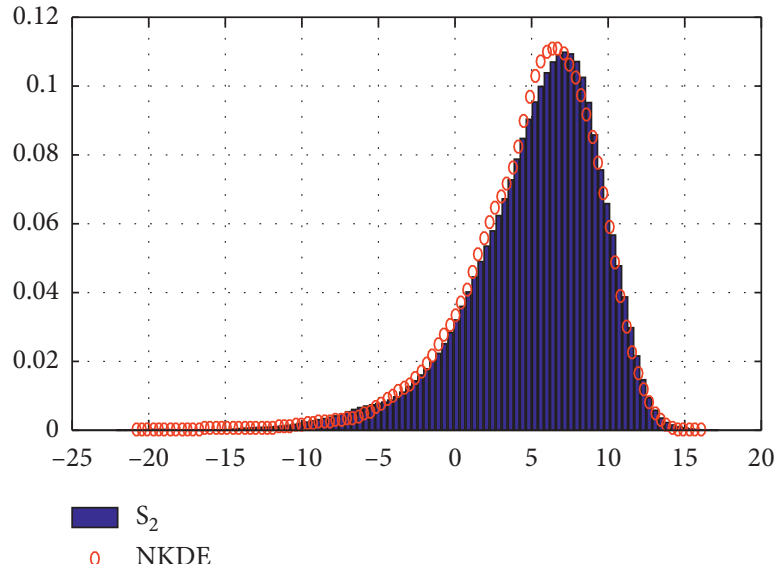

(c)

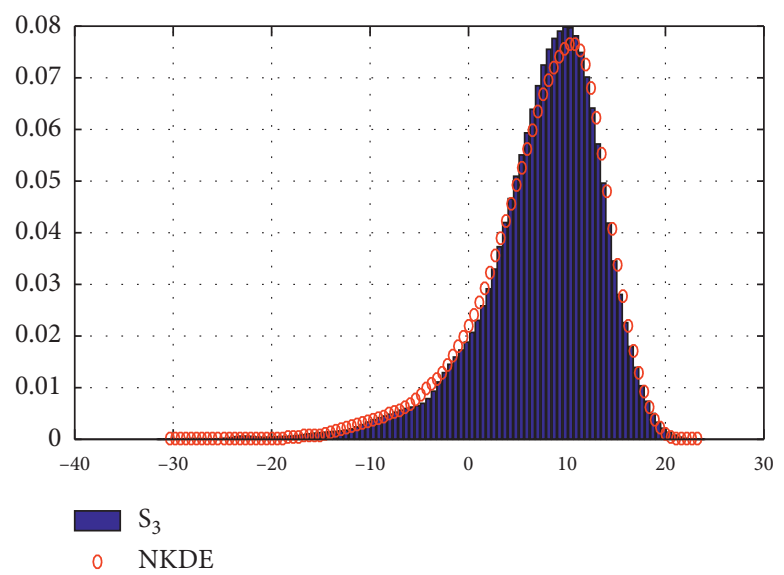

(e)

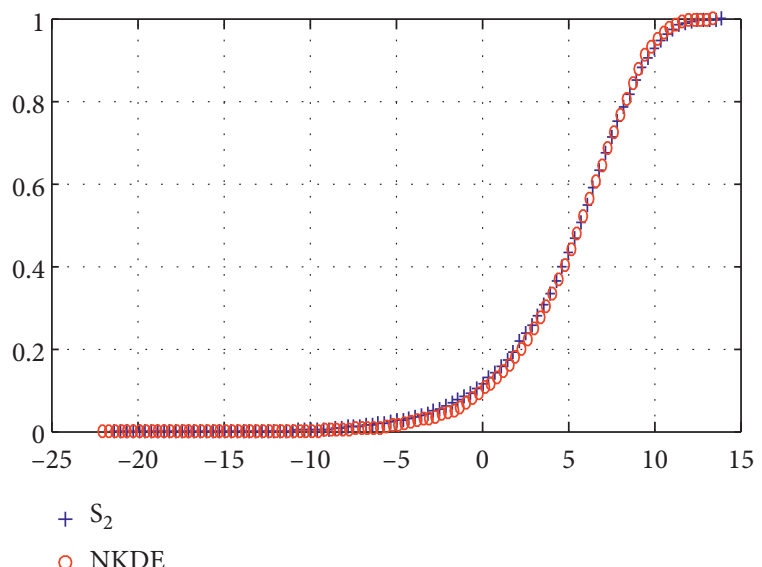

(d)

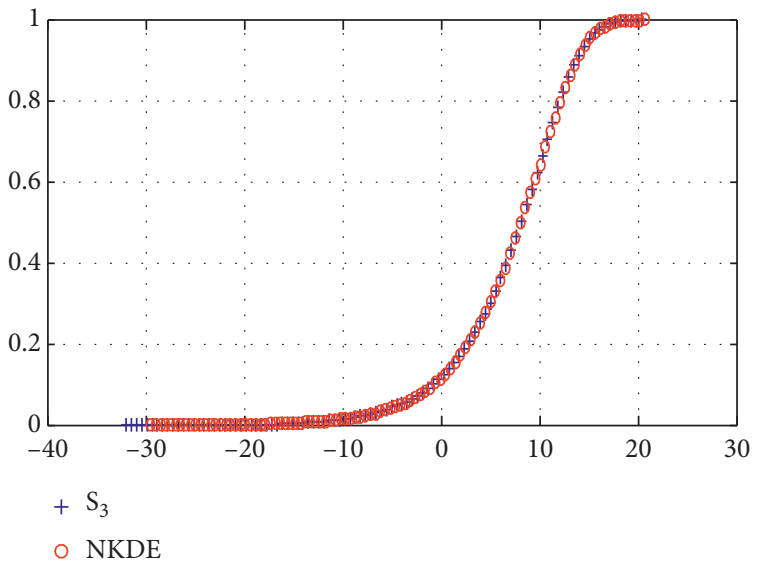

(f)

FIGURE 5: Comparison of fitting results between statistical data samples and NKDE. (a) $S_{1}$ probability density function. (b) $S_{1}$ cumulative distribution function. (c) $S_{2}$ probability density function. (d) $S_{2}$ cumulative distribution function. (e) $S_{3}$ probability density function. (f) $S_{3}$ cumulative distribution function.

TABLE 2: Estimated values of distribution parameters.

\begin{tabular}{|c|c|c|c|c|c|c|c|c|c|}
\hline \multirow{2}{*}{ Load types } & \multicolumn{3}{|c|}{$S_{1}$} & \multicolumn{3}{|c|}{$S_{2}$} & \multicolumn{3}{|c|}{$S_{3}$} \\
\hline & $\mu\left(\mathrm{kN} / \mathrm{m}^{2}\right)$ & $\sigma\left(\mathrm{kN} / \mathrm{m}^{2}\right)$ & $\delta$ & $\mu\left(\mathrm{kN} / \mathrm{m}^{2}\right)$ & $\sigma\left(\mathrm{kN} / \mathrm{m}^{2}\right)$ & $\delta$ & $\mu\left(\mathrm{kN} / \mathrm{m}^{2}\right)$ & $\sigma\left(\mathrm{kN} / \mathrm{m}^{2}\right)$ & $\delta$ \\
\hline Probabilistic characteristics & \multicolumn{3}{|c|}{ Gaussian distribution } & \multicolumn{3}{|c|}{ Gumbel I distribution } & \multicolumn{3}{|c|}{ Gumbel I distribution } \\
\hline Specimen statistics & 5.00 & 0.315 & 0.063 & 7.00 & 3.003 & 0.419 & 10.00 & 4.820 & 0.482 \\
\hline NKDE & 5.00 & 0.350 & 0.070 & 7.00 & 3.227 & 0.461 & 10.00 & 4.710 & 0.471 \\
\hline
\end{tabular}

TABLE 3: Estimated values of load distribution parameters for three groups.

\begin{tabular}{|c|c|c|c|c|c|}
\hline \multicolumn{2}{|c|}{$\begin{array}{l}\text { Probabilistic } \\
\text { characteristics } f\end{array}$} & Load $S_{1}$ & Load $S_{2}$ & Load $S_{3}$ & Bearing capacity $R$ \\
\hline \multirow{4}{*}{ Case 1} & Distribution & Gaussian distribution & Gumbel I distribution & Gumbel I distribution & Lognormal distribution \\
\hline & $\mu\left(\mathrm{kN} / \mathrm{m}^{2}\right)$ & 5 & 7 & 10 & 70 \\
\hline & $\sigma\left(\mathrm{kN} / \mathrm{m}^{2}\right)$ & 0.350 & 3.227 & 4.710 & 9.100 \\
\hline & $\delta$ & 0.070 & 0.461 & 0.471 & 0.130 \\
\hline \multirow{4}{*}{ Case 2} & Distributions & Gaussian distribution & Gumbel I distribution & Gumbel I distribution & Lognormal distribution \\
\hline & $\mu\left(\mathrm{kN} / \mathrm{m}^{2}\right)$ & 5 & 10 & 8 & 70 \\
\hline & $\sigma\left(\mathrm{kN} / \mathrm{m}^{2}\right)$ & 0.350 & 4.610 & 3.768 & 9.100 \\
\hline & $\delta$ & 0.070 & 0.461 & 0.471 & 0.130 \\
\hline
\end{tabular}


TABLE 3: Continued.

\begin{tabular}{|c|c|c|c|c|c|}
\hline \multicolumn{2}{|c|}{$\begin{array}{l}\text { Probabilistic } \\
\text { characteristics } f\end{array}$} & Load $S_{1}$ & Load $S_{2}$ & Load $S_{3}$ & Bearing capacity $R$ \\
\hline \multirow{4}{*}{ Case 3} & Distributions & Gaussian distribution & Gumbel I distribution & Gumbel I distribution & Lognormal distribution \\
\hline & $\mu\left(\mathrm{kN} / \mathrm{m}^{2}\right)$ & 5 & 7 & 10 & 70 \\
\hline & $\sigma\left(\mathrm{kN} / \mathrm{m}^{2}\right)$ & 0.350 & 3.374 & 4.630 & 9.100 \\
\hline & $\delta$ & 0.070 & 0.482 & 0.463 & 0.130 \\
\hline
\end{tabular}

Note: $S_{i}$ is the load effect value, $R$ is the bearing capacity, $\mu$ and $\sigma$ are mean and variance, respectively, and $\delta$ is coefficient of variation.

TABLE 4: Results of maximum likelihood estimation of parameters.

\begin{tabular}{lcccccc}
\hline Parameters & $\alpha$ & $\beta$ & $\gamma$ & $\omega_{1}$ & $\omega_{2}$ & $\omega_{3}$ \\
\hline Case1 & 1.4418 & 2.2433 & 6.5567 & 0.9224 & 0.0007 & 0.0769 \\
Case2 & 1.3617 & 2.2194 & 6.4877 & 0.9107 & 0.0002 & 0.0891 \\
Case3 & 1.3521 & 2.3628 & 6.3285 & 0.8826 & 0.0006 & 0.1168 \\
\hline
\end{tabular}

TABLE 5: Comparisons of reliability calculations.

\begin{tabular}{lccccc}
\hline $\begin{array}{l}\text { Combination } \\
\text { types }\end{array}$ & JCSS & Turkstra & Gong & M-Copula \\
\hline Case1 & $S$ & 38.89 & 32.91 & 32.42 & 30.83 \\
& $R_{\mathrm{el}}$ & 3.030 & 3.587 & 3.626 & 3.781 \\
\hline \multirow{2}{*}{ Case2 } & $S$ & 41.29 & 37.17 & 37.76 & 33.96 \\
& $R_{\mathrm{el}}$ & 2.86 & 3.243 & 3.213 & 3.541 \\
\hline \multirow{2}{*}{ Case3 } & $S$ & 38.45 & 33.69 & 33.93 & 30.54 \\
& $R_{\mathrm{el}}$ & 3.060 & 3.503 & 3.469 & 3.796 \\
\hline
\end{tabular}

\section{Results and Discussion}

There are many kinds of loads that contain relevance is supported during structural service, in which the load correlation would affect the results of structural reliability evaluation. The purpose of this study is to analyze the influence of load correlation on reliability. In the past research, the assumption is that the correlation among loads is always ignored, or the correlation between the two loads is considered, and it would underestimate the result of reliability evaluation and is not conducive to the balance between reliability and economy. In this paper, firstly, the probability characteristics of three groups of loads $\left(\mathrm{S}_{1}, \mathrm{~S}_{2}, \mathrm{~S}_{3}\right)$ are estimated by the NKDE method (the confidence level is $95 \%$ ). Figure 5 shows that the accuracy of NKDE fitting is very high according to the results of estimation and statistics. Secondly, the M-Copula model is proposed to build the correlation of multiple loads, and MATLAB software is used to fit the model parameters. In three numerical case studies, the M-Copula model is used to calculate reliability of different load correlation, and the results are compared with those of the JCSS, Turkstra, and Gong method. Table 5 shows that the reliability calculated by the model is the highest of the four methods. It is also found in the comparative analysis that the reliability index calculated by the M-Copula model is $24.79 \%, 23.81 \%$, and $24.05 \%$ higher than that calculated by the JCSS method in the international standard.

It can be seen that the past research methods underestimate the actual reliability, resulting in a large amount of waste of resources and adverse economic development of the structure. This study analyzes the internal relationship among loads and provides a feasible mathematical model for the economic development of reliability design. The model can be used to analyze the correlation of loads greater than 3 . However, a large number of load correlation analysis must involve multiple integration, which requires computer-aided calculation.

In this paper, the static load correlation analysis is solved. Firstly, the method of nonparametric kernel density estimation (NKDE) is used to estimate the probability density function (PDF), and a mix copula (M-Copula) function model is proposed. Finally, the correlation of related loads is obtained through MATLAB model parameter fitting. However, the dynamic load analysis of structural dynamics has some limitations, so we will carry out the study of dynamic load correlation in the future.

\section{Conclusions}

The M-Copula function model established in this paper and analysis results of specific cases can be summarized as follows:

(1) Load effect results from the combined action of multiple complex loads, which are not completely independent, and most of the loads are correlated. Previous studies have only considered that loads are independent of each other, not related to each other, or that correlation between two loads is considered; obviously, these are unreasonable.

(2) Loads are random variables, and in fact they cannot be completely decoupled and independent. The NKDE method can be used to fit three kinds of related loads, and results show that the method has high accuracy.

(3) Establishing the M-Copula function model can accurately describe the relationship between loads and calculate correlation between loads through Gumbel copula, Frank copula, Clayton copula function, and comprehensive model of weight coefficient. By analyzing actual data of three kinds of related loads, the results show that M-Copula function has a high consistency in estimating correlation between multivariate loads.

(4) Comparing the results of the reliability index calculated by the M-Copula model with those calculated by the traditional JCSS method and Turkstra method, it is found that the calculation method used 
in the past is too conservative, which is not conducive to the result of structural performance evaluation and economy of later structural treatment, while the M-Copula model accurately calculates load effects, and current performance should be accurately evaluated by combining the values and structural reliability indicators.

\section{Notations}

$\begin{array}{ll}S: & \text { Load effect } \\ F(\cdot): & \text { Joint distribution function } \\ C(\cdot): & \text { Copula function } \\ f(\cdot): & \text { Joint probability density function } \\ \Phi(\cdot): & \text { Standard Gauss distribution } \\ & \text { function } \\ \phi_{\alpha}(\cdot), \phi_{\beta}(\cdot), \text { and } \phi_{\gamma}(\cdot): & \text { Generators of Gumbel, Frank, and } \\ \alpha, \beta, \text { and } \gamma: & \text { Clayton function } \\ C^{G}, C^{F}, \text { and } C^{C}: & \text { Parameters of copula function } \\ & \text { Copula function of Gumbel, Frank, } \\ C_{\mathrm{mix}}(\cdot): & \text { and Clayton } \\ \omega: & \text { Mix copula function } \\ h_{n}: & \text { Weight coefficient } \\ K(\cdot): & \text { Bandwidth } \\ \rho(\cdot): & \text { Kernel function } \\ R: & \text { Kendall's correlation coefficient } \\ \mu: & \text { Bearing capacity } \\ \sigma: & \text { Mean value } \\ \delta: & \text { Standard deviation } \\ R_{\mathrm{el}}: & \text { Coefficient of variation }\end{array}$

\section{Data Availability}

All data generated or analyzed during this study are included in this article.

\section{Conflicts of Interest}

The authors declare that they have no conflicts of interest.

\section{Acknowledgments}

This work was supported by the National Key Fund (51890903) and the National Key R\&D Program of China Grant (grant numbers 2016YFC 0701301-01)-Probabilistic model and time-varying reliability of the complex environmental effects of industrial buildings.

\section{Supplementary Materials}

The specimen statistics are provided as the supplementary materials. (Supplementary Materials)

\section{References}

[1] X. Zhang, H. Gao, H.-Z. Huang, Y.-F. Li, and J. Mi, "Dynamic reliability modeling for system analysis under complex load," Reliability Engineering \& System Safety, vol. 180, pp. 345-351, 2018.
[2] H. Ma, Y. Yang, Z. He, Z. Jia, and Y. Zhang, "Experimental study on constitutive relation of the high performance marine structural steel under extreme cyclic loads," Ocean Engineering, vol. 168, pp. 204-215, 2018.

[3] B. Kim and J. Y. Lee, "Resistance of interfacial debonding failure of GFRP bars embedded in concrete reinforced with structural fibers under cycling loads," Composites Part B-Engineering, vol. 156, pp. 201-211, 2019.

[4] K. M. C. Konthesingha, M. G. Stewart, P. Ryan, J. Ginger, and D. Henderson, "Reliability based vulnerability modelling of metal-clad industrial buildings to extreme wind loading for cyclonic regions," Journal of Wind Engineering and Industrial Aerodynamics, vol. 147, pp. 176-185, 2015.

[5] A. Vaghefi, F. Farzan, and M. A. Jafari, "Modeling industrial loads in non-residential buildings," Applied Energy, vol. 158, pp. 378-389, 2015.

[6] F. Scozzese, G. Terracciano, A. Zona, G. Della Corte, A. Dall'Asta, and R. Landolfo, "Analysis of seismic nonstructural damage in single-storey industrial steel buildings," Soil Dynamics and Earthquake Engineering, vol. 114, pp. 505-519, 2018.

[7] Y. K. Wen, "Statistical combination of extreme loads," Journal of the Structural.Division, vol. 103, no. 5, pp. 1079-1093, 1977.

[8] K. Breitung and R. Rackwitz, "Nonlinear combination of load processes," Journal of Structural Mechanics, vol. 10, no. 2, pp. 145-166, 1982.

[9] J. X. Gong and G. F. Zhao, "Combination of relevant load effects and reliability calculation," Journal of Engineering Mechanics, vol. 18, no. 4, pp. 1-6, 2001.

[10] Q. W. Li and C. Wang, "Effect of load random process relevance on structural time-varying reliability," Journal of .Tsinghua University: Natural Science Edition, vol. 54, no. 10, pp. 1316-1320, 2014.

[11] J. T. Yao, "Time-interval analysis method for structural reliability," Journal of Civil Engineering, vol. 10, no. 7, pp. 1-5, 2005.

[12] Z. M. Lin, Reliability Design and Evaluation of Engineering Structures, People's Communications Publishing House, Beijing, China, 1990.

[13] M. Dupraz, S. I. Rao, and H. Van Swygenhoven, "Large scale 3-dimensional atomistic simulations of screw dislocations interacting with coherent twin boundaries in $\mathrm{Al}, \mathrm{Cu}$ and $\mathrm{Ni}$ under uniaxial and multiaxial loading conditions," Acta Materialia, vol. 174, pp. 16-28, 2019.

[14] A. Sklar, Fonctions de repartition a $n$ dimensions et leurs marges, Publications De I'Institut De Statistique De L'Uinversite De Paris, Paris, France, 1959.

[15] U. Cherubini, E. Luciano, and Vecchiato, Copula Methods in Finance, John Wiley \& Sons, Chichester, England, 2004.

[16] S. Ghosh, "Modelling bivariate rainfall distribution and generating bivariate correlated rainfall data in neighbouring meteorological subdivisions using copula," Hydrological Processes, vol. 24, no. 24, pp. 3558-3567, 2010.

[17] Y. Cai, J. Cai, L. Xu, Q. Tan, and Q. Xu, "Integrated risk analysis of water-energy nexus systems based on systems dynamics, orthogonal design and copula analysis," Renewable and Sustainable Energy Reviews, vol. 99, pp. 125-137, 2019.

[18] S. Chen, J. Xu, Q. Li, X. Tan, and X. Nong, “A copula-based interval-bistochastic programming method for regional water allocation under uncertainty," Agricultural Water Management, vol. 217, pp. 154-164, 2019.

[19] K. Goda, "Statistical modeling of joint probability distribution using copula: application to peak and permanent 
displacement seismic demands," Structural Safety, vol. 32, no. 2, pp. 112-123, 2010.

[20] B. J. Leira, "Probabilistic assessment of weld fatigue damage for a nonlinear combination of correlated stress components," Probabilistic Engineering Mechanics, vol. 26, no. 3, pp. 492500, 2011.

[21] X.-S. Tang, D.-Q. Li, C.-B. Zhou, K.-K. Phoon, and L.-M. Zhang, "Impact of copulas for modeling bivariate distributions on system reliability," Structural Safety, vol. 44, pp. 80-90, 2013.

[22] C. Jiang, W. Zhang, B. Wang, and X. Han, "Structural reliability analysis using a Copula-function-based evidence theory model," Computers \& Structures, vol. 143, pp. 19-31, 2014.

[23] J. Zhang, X. Ma, and Y. Zhao, "A stress-strength time-varying correlation interference model for structural reliability analysis using Copulas," IEEE Transactions on Reliability, vol. 66, no. 2, pp. 351-365, 2017.

[24] H. An, H. Yin, and F. He, "Analysis and application of mechanical system reliability model based on Copula function," Polish Maritime Research, vol. 23, no. s1, pp. 187-191, 2016.

[25] R. Lebrun and A. Dutfoy, "A generalization of the Nataf transformation to distributions with elliptical copula," Probabilistic Engineering Mechanics, vol. 24, no. 2, pp. 172178, 2009.

[26] Z. B. Tu, M. F. Huang, and W. J. Lou, "Correlation of wind loads on building dynamic based on Copula function," Journal of Zhejiang University-Science, vol. 48, no. 8, pp. 1370-1375, 2014.

[27] S. B. Hu, Z. L. Jiang, and X. R. Zhu, "Research on Parzen window based on improve Gaussian matrix in medical image registration," Journal of Computational Information System, vol. 8, no. 12, pp. 5103-5110, 2012.

[28] A. Gramacki, Nonparametric Kernel Density Estimation and its Computational Aspects, Springer, New York, NY, USA, 1st edition, 2018.

[29] A. M. Elgammal, Efficient Nonparametric Kernel Density Estimation for Real Time Computer Vision, University of Maryland at College Park, College Park, MD, USA, 2002.

[30] Z. Qin, W. Li, and X. Xiong, "Estimating wind speed probability distribution using kernel density method," Electric Power Systems Research, vol. 81, no. 12, pp. 2139-2146, 2011.

[31] K. Choi and J. Marden, "An approach to multivariate rank tests in multivariate analysis of variance," Journal of the American Statistical Association, vol. 92, no. 440, pp. 15811590, 1997. 